\title{
Il muro improbabile. Alcune considerazioni sul film Dogtooth
}

La storia raccontata in Dogtooth, probabilmente debitrice di alcune ispirate pellicole della storia del cinema (L'angelo sterminatore di Buñuel e $I l$ castello della purezza di Arturo Ripstein) è semplice: un padre di famiglia decide di relegare e crescere i propri figli e la moglie in casa, isolandoli completamente dal resto del mondo. Spiegherà ai figli che potranno uscire di casa solo quando uno dei denti canini cadrà e ricrescerà (come racconta essere accaduto a lui), segno biologico della sopraggiunta maturità necessaria per affrontare il mondo.

Dovendo cimentarmi col tentativo di proporre una lettura del film, l'impresa si è rivelata subito per ciò che era: complicata. Ma provo a spiegarmi.

A fronte, infatti, dell'apparente semplicità della metafora scelta e dei fatti narrati - pochi -, il regista decide di mettere in scena un quadro di schegge e pezzi difficile da tenere insieme.

La difficoltà incontrata a connettere i 'pezzi' in un insieme coerente narrativo e visivo (effetto ottenuto grazie a un sapiente montaggio e uso della videocamera), ovviamente lungi dall'essere un fallimento del plot narrativo, ci racconta in realtà di come il film sappia cogliere nel segno, imponendo allo spettatore di fare esperienza della frammentazione come uno tra gli effetti auspicati da ogni regime totalitario, di cui il film ci offre una rappresentazione metaforica. E cioè: l'effetto di straniamento, la difficoltà a riconnettere le inquadrature, le scene, le sequenze entro una narrazione coerente e stabile è ciò che ci accomuna ai protagonisti della storia, mettendo in campo un processo parallelo in cui la confusione e l'angoscia, scanditi entro un ritmo di montaggio sincopato e imprevedibile, sono gli stati d'animo dominanti proprio di chi (spettatore e protagonisti) non ha in mente il quadro generale, il senso, il progetto, di cui è titolare esclusivo e capriccioso detentore il Padre.

\footnotetext{
*Psicologo, Psicoterapeuta Transculturale a Milano; Supervisore in ambito clinico e socio-educativo; Sceneggiatore, formatore in metodologie audiovisive; Referente del progetto "Psicologia e Cinema" per OPL (Ordine Psicologi Lombardia).

E-mail: alessandrociardi5@gmail.com
} 
Ogni forma di dittatura fonda il proprio status e potere sulla deprivazione e manipolazione degli stimoli e delle informazioni, alterando la percezione della realtà. Il film mette in scena proprio gli effetti psichici e fisici di una forma estrema e radicale di potere, che articola e struttura un ingranaggio e dei dispositivi (ma come ogni macchina, fallibile...) tesi al controllo dei comportamenti, a partire dal controllo del linguaggio - quindi, del pensiero. Il regista sceglie una chiave allegorica, affidando lo svolgimento della narrazione a un dramma con forte impianto teatrale, che si svolge per lo più tra le mura domestiche.

Si è da subito tentati di pensare che lo sguardo e la critica del regista possa alludere alla nostra contemporaneità occidentale, nella quale certamente facciamo costante esperienza della libertà ma, come sostengono alcuni, in una sua forma sempre più perversa e pervertita (si pensi alla profetica critica pasoliniana sulla società dei consumi o sugli effetti del potere sui corpi; alle tesi di Debord sulla società dello spettacolo, sino al pensiero del filosofo Agamben, pur nella sua discutibile radicalità, quando scrive del campo di concentramento come la verità del progetto politico occidentale).

Il primo oggetto inquadrato, all'inizio del film, è un registratore, di cui ascoltiamo la voce monotona - già questo basti a disorientare lo spettatore, che ancor prima di vedere, ascolta, come un infante, che è poi la condizione cui sono relegati e in cui sono abbandonati i protagonisti della storia.

La voce registrata nomina alcune parole e il loro significato. Questa prima scena si svolge in un bagno, dove due ragazze e un ragazzo, che scopriremo essere sorelle e fratello, ascoltano e seguono con attenzione la lezione (la scelta di una location bizzarra, letteralmente fuori luogo per una attività di studio, rimarca nello spettatore lo straniamento). Salvo scoprire - ma è una consapevolezza dello spettatore, non dei personaggi, secondo un classico meccanismo narrativo - che ai significanti elencati vengono attribuiti dei significati alterati, arbitrari (laddove, ad esempio, 'mare' è una poltrona). Questo prologo esprime già chiaramente lo stile del film: lo scardinamento delle aspettative nella scelta dell'ambiente, le inquadrature fisse (sia su chi parla sia alcuni estenuanti piani di ascolto sul personaggio), la recitazione meccanica, impersonale delle attrici e dell'attore, la perversione del linguaggio - che significherà perversione incestuosa dei legami e dell'ordine tra generazioni -.

I personaggi, schiavi senza sapere di esserlo, asserviti al capriccio del persecutore/salvatore, si vedono inibiti nella naturale propensione all'esplorazione - del mondo e di sé -, all'autoaffermazione e autodeterminazione, riducendosi ad automi ed ingranaggi che eseguono i compiti attribuiti affinché il progetto e la macchina sopravvivano, nell'espressione compiaciuta dell'autorità. Il ragazzo e le ragazze, anagraficamente ormai 
adulti, si muovono nello spazio e interagiscono tra loro in modo goffo e maldestro, come bambini deprivati di stimoli, resi incapaci di motilità fine e di consapevolezza di sé nello spazio.

La fotografia essenziale, la messa in scena scarna si articolano in inquadrature fisse, entro le quali continuano ad alternarsi figure intere e volti a dettagli di corpi, rendendo impossibile allo spettatore di ricomporre i personaggi entro quadri comprensibili, in un 'intero' anatomico - giocando, come si fa sempre più raramente, a sovvertire le aspettative dello spettatore, fin troppo abituato a una estetica cinematografica prevedibile e un po' compiaciuta. La telecamera infatti, sembra costantemente fare e disfare corpi, smontando e rimontando pezzi, come una tela di Penelope, aumentando l'inquietudine dello spettatore che arranca nella ricomposizione di un intero in cui mancano pezzi o vengono costantemente rimescolati da capo - infondendo in chi guarda una sensazione di caos e angoscia.

Il padre è l'unico che esce di casa - e, nel tentativo di perpetuare l'inganno, si adopera, con il sostegno della moglie, a mettere in campo numerosi artifici: dai pesci comprati e ributtati in piscina sino alla traduzione simultanea di una canzone di Sinatra, ascoltata dopo cena (spacciato ai ragazzi per il loro nonno!), che andrebbe cantando la necessità di un ordine morale e di una disciplina improntata al rispetto delle regole e dell' indiscutibile autorità genitoriale.

Alcune delle recensioni del film hanno rilevato come il regista, attraverso la messa in scena di questa famiglia e di questo padre, stia alludendo al ruolo dello Stato. La questione andrebbe forse meglio collocata e riformulata precisando che questa messa in scena rappresenti una forma perversa del potere e della Legge del padre. Insomma, un padre che si crede un Padre commette un errore educativo fatale. E, nella versione proposta dal regista, il padre incarna il volto di una legge morale stravolta che non ammette insubordinazioni, fossero anche semplicemente i movimenti di separazione/individuazione dei figli, per il compimento dei quali è necessario tradire, come ci ricorda Aldo Carotenuto.

L'attribuzione arbitraria tra significato e significante, che a partire dalla scena iniziale, sarà colonna portante di tutto l'impianto del film, sembra farsi portatrice, indirettamente, di un tema: da un lato la necessità e il bisogno di una regola comune non solo utile al patto sociale ma anche all'equilibrio psichico - penso qui alla definizione di E. Minkowski di psicosi come estensione anarchica del campo dei significati. Dall'altro, tuttavia, viene posta anche la questione dell'arbitrarietà delle regole - per la serie: chi decide che al significante $x$ debba corrispondere il significato $y$ e non $z$ ?

Tuttavia, anche se questa versione del padre - che tende appunto alla père-version - rappresenta il tentativo di costruire un universo privo di 
falle, capace di saturare il senso e qualsiasi tipo di mancanza, come ogni dispositivo, ad un tratto, può incrinarsi e da quella incrinatura può finalmente intrufolarsi e fare breccia il dubbio, la mancanza e quindi, il desiderio.

Un rapporto di scambio, infatti, rende complici una delle figlie e una ragazza (che viene condotta dal padre sino alla casa bendata e che viene introdotta tra le mura domestiche per consentire al figlio di avere rapporti sessuali). Dalla frequentazione della casa, da parte di questa 'esterna', scaturirà un rapporto di complicità con una delle sorelle: quest'ultima, infatti, ottiene dalla ragazza, fornendo favori sessuali, alcuni oggetti - per altro, privi di valore. Ma, ad un certo punto la sorella chiede che la ragazza le lasci due Videocassette. E saranno proprio queste due videocassette a introdurre nuovi significati e a gettare le prime ombre sull'universo totalitario messo in scena dal padre - curiosamente proprio due film, due opere di finzione introducono la verità nel castello dorato. Sembrerebbe qui che il regista stia alludendo a una funzione del cinema e dell'arte in generale di assolvere al compito di fare breccia entro sistemi di credenze e pregiudizi per portare il beneficio del dubbio, ricordandoci che un sistema sano - istituzioni comprese - sa e deve abbracciare la propria complessità, scansando le derive di facili e seducenti ipersemplicazioni.

Va detto, per completezza, che il film mette in scena anche il mondo di fuori - il padre lavora in una azienda. Ben lontano dall'essere violento e infido, come forse viene descritto ai figli, il mondo esterno è di una ordinarietà e grigiore sconfortanti. Queste caratteristiche del mondo esterno, lasciato sullo sfondo e a margine della narrazione, rappresentano, credo, un punto centrale - che è anche cifra stilistica del film, laddove in molte inquadrature compaiono porzioni di spazio disabitate, assolutamente anonime, della casa. Il meccanismo della perversione paterna è reso qui più estremo e paradossale proprio perché all'esterno non c'è alcun pericolo o minaccia - se non quello, forse, di assuefarsi a una vita insignificante e di morire, un giorno alla volta.

Dunque, il regista e lo sceneggiatore lavorano sulle singole scene nello stesso modo in cui lavorano con la struttura drammaturgica in generale, seguendo il principio secondo cui ciò che è inquadrato e ritenuto importante potrebbe non esserlo quanto ciò che non è inquadrato e reso periferico.

Credo che questo rappresenti un potente spunto di riflessione sulla contemporaneità, un invito politico a continuare a tenere lo sguardo anche e soprattutto sui/ai margini, laddove potrebbero accadere rivoluzioni o involuzioni senza che ce ne accorgiamo, accecati da ciò che, nella sua luminosa vacuità, si impone al centro della scena e ci distrae da ciò che conta davvero.

E ciò mi porta a una ultima considerazione - qui, ripeto, risiede forse 
un messaggio politico più nascosto del film: il compito degli adulti di oggi per le future generazioni non può più consistere nel creare spazi esclusivi, immunizzati e difesi - se mai ciò ha avuto senso -, ma deve articolare tutti gli sforzi possibili per rendere il mondo un posto più bello e giusto da abitare per una vita più degna da vivere.

Conflitto di interessi: l'autore dichiara che non vi sono potenziali conflitti di interessi.

Approvazione etica e consenso a partecipare: non necessario.

Ricevuto per la pubblicazione: 8 dicembre 2020 .

Accettato per la pubblicazione: 24 gennaio 2021.

${ }^{\circ}$ Copyright: the Author(s), 2021

Licensee PAGEPress, Italy

Ricerca Psicoanalitica 2021; XXXII:500

doi:10.4081/rp.2021.500

This article is distributed under the terms of the Creative Commons Attribution Noncommercial License (by-nc 4.0) which permits any noncommercial use, distribution, and reproduction in any medium, provided the original author(s) and source are credited. 
